\title{
The Resistivity of Zinc Oxide Under Different Annealing Configurations and Its Impact on the Leakage Characteristics of Zinc Oxide Thin-Film Transistors
}

\author{
Lei Lu and Man Wong, Senior Member, IEEE
}

\begin{abstract}
Sputtered zinc oxide ( $\mathrm{ZnO}$ ) without intentional doping was thermally annealed and the dependence of its resistivity on different sample configurations and heat-treatment conditions was studied. The $\mathrm{ZnO}$ was either exposed to the ambience or sealed with an impermeable cover during the annealing. The resistivity resulting from the sealed configuration was found to be lower. The possible origins of the charge carriers responsible for the conductivity were investigated, with the most plausible one studied in greater detail using photoluminescence. The leakage current in the OFF-state of a field-effect thin-film transistor is largely controlled by the residual conductivity of the channel region of the transistor. For a $\mathrm{ZnO}$ transistor, this region is typically undoped and subjected to a series of thermal processes under a variety of coverage configurations during the course of the fabrication of the transistor. The implied correlation between the aggregate thermal treatment and the characteristics of a transistor in its OFF-state was investigated and demonstrated.
\end{abstract}

Index Terms-Defects, photoluminescence (PL), resistivity, silicon nitride, silicon oxide, thermal annealing, thin-film transistors (TFT), zinc oxide ( $\mathrm{ZnO})$.

\section{INTRODUCTION}

$\mathbf{Z}$ INC oxide $(\mathrm{ZnO})$ and its variants are being pursued for a host of applications, including solar cell, lightemitting diode, transparent conductor, and thin-film transistor (TFT) [1], [2]. ZnO-based TFT is a promising candidate for replacing silicon-based TFT in flat-panel displays, because of its higher transparency within the visible spectrum, higher ON-OFF current ratio, higher field-effect mobility, and lower processing temperature [3], [4].

The properties of $\mathrm{ZnO}$, such as crystallinity [5], [6], resistivity [6]-[9], and photoluminescence (PL) [9]-[12] are sensitive to how the $\mathrm{ZnO}$ is thermally processed. In the making of a $\mathrm{ZnO}$ TFT, thermal processing is unavoidable for meeting a variety

Manuscript received November 9, 2013; revised January 8, 2014; accepted January 22, 2014. Date of publication February 11, 2014; date of current version March 20, 2014. This work was partially supported by the Partner State Key Laboratory on Advanced Displays and Optoelectronics Technologies under Grant ITC-PSKL12EG02. The review of this paper was arranged by Editor J. Huang.

The authors are with the Department of Electronic and Computer Engineering, the Hong Kong University of Science and Technology, Hong Kong (e-mail. luleiece@ust.hk; eemwong@ece.ust.hk).

Color versions of one or more of the figures in this paper are available online at http://ieeexplore.iee.org.

Digital Object Identifier 10.1109/TED.2014.2302431 of requirements. These include stabilizing [8] and improving the crystallinity [13], [14] of the $\mathrm{ZnO}$ film, decreasing the contact resistance [15], and activating the dopants in the $\mathrm{ZnO}$ [16], [17]. If the properties of the channel region of a TFT should change during the course of these thermal processes, the characteristics of the transistor would also be affected. One example is the effects of the conductivity of the channel region on the leakage current of the transistor in its OFF-state.

Hitherto most of the studies [5]-[12] on the effects of the thermal annealing of $\mathrm{ZnO}$ were performed on bare samples. This does not reflect the reality of a TFT [1], [2], the channel region of which is typically covered either by the gate dielectric or by a passivation layer. Popular cover-layers are silicon oxide $\left(\mathrm{SiO}_{x}\right)$ or silicon nitride $\left(\mathrm{SiN}_{x}\right)$ [1], [2], [8], [13]-[17]. The annealing behavior of such covered $\mathrm{ZnO}$ may be different from that of a bare $\mathrm{ZnO}$, perhaps also dependent on the permeability of the cover.

The dependence of the resistivity $\rho$ of sputtered $\mathrm{ZnO}$ thin films on the annealing ambience, time, and temperature were systematically investigated for bare $\mathrm{ZnO}, \mathrm{ZnO}$ with a permeable cover and $\mathrm{ZnO}$ with an impermeable seal. The $\rho$ of the last configuration was found to be the lowest. The possible origins of the charge carriers responsible for the conductivity were investigated, with the most plausible one further studied using PL.

The OFF-state leakage current of a field-effect TFT is largely controlled by the residual conductivity of the channel region of the transistor. This region is typically undoped for a $\mathrm{ZnO}$ TFT and, during the course of fabrication of the transistor, subjected to a series of thermal processes under a variety of coverage configurations. The implied correlation between the aggregate thermal treatment and the leakage current was investigated and demonstrated.

\section{Heat-Treatment of ZnO With DifFerent TYPES OF COVER FILMS}

\section{A. Dependence of the $\rho$ on the Heat-Treatment Ambience}

Three kinds of samples were investigated for comparison. These were Sample B: bare $\mathrm{ZnO}$; Sample O: $\mathrm{ZnO}$ covered with $\mathrm{SiO}_{x}$; and Sample ON: $\mathrm{ZnO}$ covered first with a layer of $\mathrm{SiO}_{x}$, then capped with a layer of $\mathrm{SiN}_{x}$. The preparation of the samples started with a room-temperature deposition 


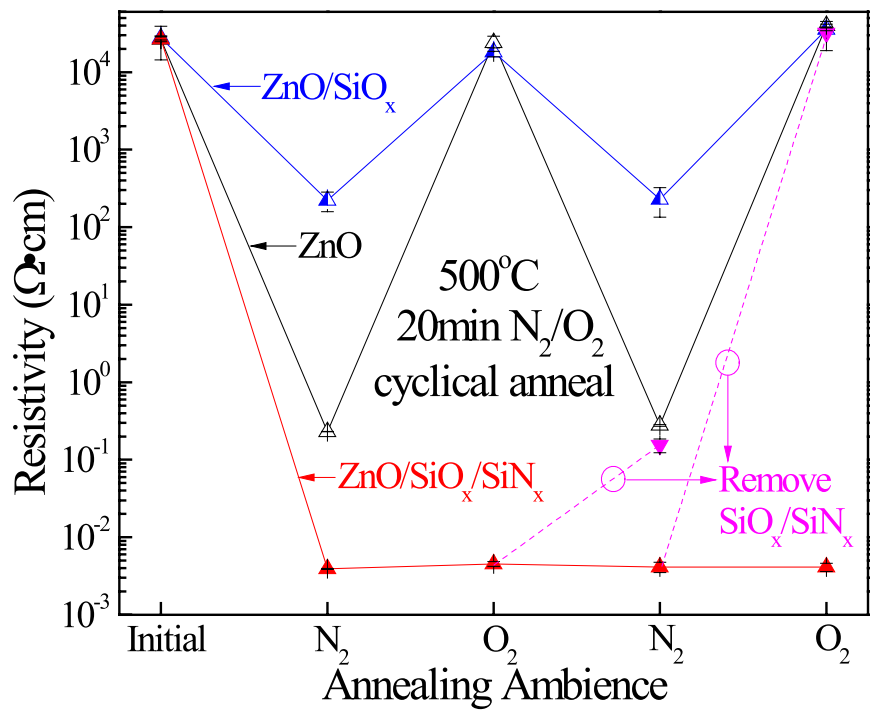

Fig. 1. Dependence of $\rho$ on the annealing ambience for Samples B, $\mathrm{O}$, and $\mathrm{ON}$.

of $100-\mathrm{nm} \mathrm{ZnO}$ on thermally oxidized silicon wafers in a 13.56- $\mathrm{MHz}$ radio frequency magnetron sputtering machine. The thickness of the thermal oxide was $500 \mathrm{~nm}$. The sputtering ambience was $10 \%$ oxygen $\left(\mathrm{O}_{2}\right)$ and $90 \%$ argon $(\mathrm{Ar})$, with a total pressure of 5 mtorr, a power density of $6 \mathrm{~W} / \mathrm{cm}^{2}$ and a target-to-substrate separation of $15 \mathrm{~cm}$. For Sample O, a layer of 100-nm thick $\mathrm{SiO}_{x}$ was subsequently deposited at $300{ }^{\circ} \mathrm{C}$ in a plasma-enhanced chemical vapor deposition (PECVD) reactor using tetraethylorthosilicate (TEOS) and $\mathrm{O}_{2}$ as the source gases. For Sample ON, an additional 200-nm $\mathrm{SiN}_{x}$ was deposited at $300{ }^{\circ} \mathrm{C}$ in a different PECVD reactor using silane and ammonia $\left(\mathrm{NH}_{3}\right)$ as the source gases.

The $\rho$ of Sample B was directly measured using a four-point probe setup, while the Samples $O$ and ON were tested after the cover-layers were removed in a reactive-ion etcher running a chemistry based on sulfur hexafluoride $\left(\mathrm{SF}_{6}\right)$. The etching process did not affect the $\rho$ of Samples $\mathrm{O}$ and $\mathrm{ON}$, as inferred from the unchanged resistivity of a simultaneously etched unannealed Sample B. All samples exhibited n-type conductivity, confirmed using Hall Effect measurements. The influence of the annealing ambience on the $\rho$ (Fig. 1) was investigated by subjecting the samples to 20 -min isochronal heat-treatments at $500{ }^{\circ} \mathrm{C}$ in alternating ambience of $\mathrm{O}_{2}$ or nitrogen $\left(\mathrm{N}_{2}\right)$.

Consistent with the findings of the previous reports [6]-[9], the $\rho$ of Sample B was observed to change with the cyclical change in the ambience, with the $\rho$ after annealing in $\mathrm{O}_{2}$ about $10^{5}$ times larger than that annealed in $\mathrm{N}_{2}$. Since the sample had not been intentionally doped, the charge-carriers responsible for the conductivity after annealing in $\mathrm{N}_{2}$ most likely originated from donor-like defects [6]-[9], [18], [19] (hereafter denoted simply as defects), such as oxygen vacancies $\left(V_{\mathrm{O}}\right)$, zinc interstitials $\left(\mathrm{Zn}_{\mathrm{i}}\right)$, or zinc antisites $\left(\mathrm{Zn}_{\mathrm{O}}\right)$ [9], [18]-[22], which were formed during the annealing in the $\mathrm{O}_{2}$-deficient $\mathrm{N}_{2}$ ambience. These defects were annihilated upon annealing in an $\mathrm{O}_{2}$ ambience, thus recovering the highly resistive state.

For Sample $\mathrm{O}$, the $\rho$ also changed cyclically with the annealing ambience, indicating there was also an exchange of oxygen-carrying species (hereafter denoted simply as species) between the ambience and the $\mathrm{ZnO}$ through the $\mathrm{SiO}_{x}$ cover. However, the ratio of the $\rho$ between Sample $\mathrm{O}$ annealed in $\mathrm{O}_{2}$ and $\mathrm{N}_{2}$ was only $\sim 10^{2}$, much smaller than the $\sim 10^{5}$ measured on Sample B. It can be concluded that either 1) the $\mathrm{SiO}_{x}$ cover layer hindered but did not prevent the exchange of the species between the ambience and the $\mathrm{ZnO}$ or 2) the $\mathrm{SiO}_{x}$ layer was itself a finite source or sink of such species. Both possibilities could lead to a smaller ambience-induced change in the $\rho$.

On the contrary, the $\rho$ of Sample ON maintained a low value and was insensitive to the cyclical change of the ambience. Consequently, the $\mathrm{SiO}_{x} / \mathrm{SiN}_{x}$ double-layer could be considered an impermeable seal and was capable of completely preventing the exchange of the species between the $\mathrm{ZnO}$ and the ambience. The $\rho$ of the thermally annealed Sample ON, being $\sim 10^{2}$ times smaller than that of Sample B annealed in $\mathrm{N}_{2}$, is an indication that either the mechanisms or the rates of the formation of the defects in the two kinds of samples are not entirely identical.

When the double-layer cover on Sample ON was removed after a heat-treatment and the exposed sample was subsequently annealed in $\mathrm{O}_{2}$, the resulting $\rho$ was the same as that of Sample B annealed in $\mathrm{O}_{2}$, as indicated by the dashed line in Fig. 1. This shows that the donors in Sample ON, like those in Sample B, can also be eliminated by an oxidizing annealing.

\section{B. Dependence of the $\rho$ on the Heat-Treatment Time}

At an annealing temperature of $500{ }^{\circ} \mathrm{C}$ in $\mathrm{N}_{2}$, the time evolution of the $\rho$ of Samples B and ON (Fig. 2) was similar, each showing a quick initial drop before reaching a steady-state value beyond a corresponding characteristic time $\left(t_{\mathrm{ss}}\right)$. For Sample B, this process consists of the loss of oxygen from $\mathrm{ZnO}$, leading to the formation of defects. For Sample ON, the presence of the impermeable seal prevented the exchange of the oxygen-carrying species between the $\mathrm{ZnO}$ and the ambience. The absence of the need for the transport of such species could be the reason for the faster kinetics, hence a shorter $t_{\mathrm{ss}}$, for Sample ON. It was further observed that the steady-state $\rho$ of Sample ON was $\sim 10^{2}$ smaller than that of Sample B. It is clear that the configuration ON is more efficient in turning intrinsic $\mathrm{ZnO}$ into a conductor, achieving a lower steady-state $\rho$ after a shorter $t_{\mathrm{ss}}$.

The behavior of the $\rho$ of Sample $\mathrm{O}$ is rather different from that of Samples B and ON, with a relatively flat plateau appearing between a more rapid initial drop for annealing time within the first $5 \mathrm{~min}$ and a gradual decrease for annealing time beyond $\sim 40 \mathrm{~min}$. If the cover-oxide were permeable but only retarded the transport of the species, then Sample O should behave like Sample B albeit with a stretched-out dependence of $\rho$ on the heat-treatment time. On the other hand, if the cover-oxide were an impermeable seal against the transport of the species, then Sample O should behave like Sample ON. Neither of these was observed.

It is presently proposed that the cover-oxide served as a finite source and sink of certain oxidizing agents (hereafter denoted simply as agents), which might or might not be 


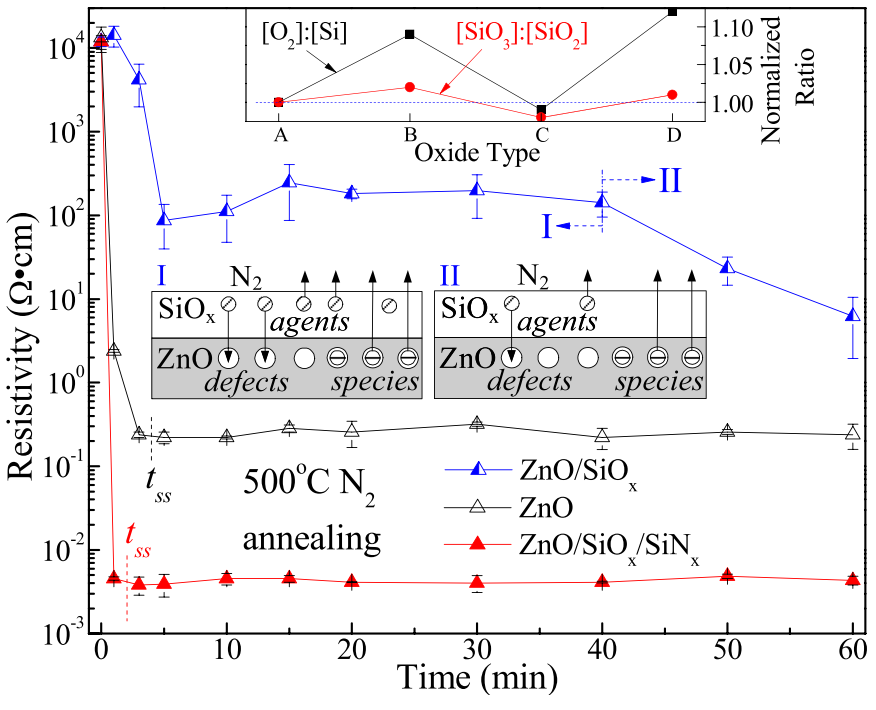

Fig. 2. Dependence of the $\rho$ on the heat-treatment time for Samples B, O, and $\mathrm{ON}$. Shown in the inset at the top are the normalized ratios of $\left[\mathrm{O}_{2}\right]:[\mathrm{Si}]$ and $\left[\mathrm{SiO}_{3}\right]:\left[\mathrm{SiO}_{2}\right]$ for the four types of silicon oxide samples.

the same as the oxygen-carrying species from the ambience. These agents, once released by the oxide, would diffuse either to the top surface of the oxide and escape to the ambience or to the bottom oxide/ $\mathrm{ZnO}$ interface and enter the $\mathrm{ZnO}$. The fraction that entered the $\mathrm{ZnO}$ oxidized, hence annihilated, some of the generated defects, thus explaining the relatively higher value of the $\rho$ than that of Sample B. A relatively flat plateau was reached when a balance was achieved between the generation and the annihilation rates of the defects (inset I of Fig. 2). Beyond 40-min annealing, the cover-oxide was gradually depleted of the oxidizing agents and the $\rho$ resumed its reduction due to uncompensated defect generation (inset II of Fig. 2), albeit at a rate slower than that of the initial drop in the $\rho$ of Sample B due to the retarded transport of the species across the oxide cover layer. It is reasonable to expect that upon an extended heat treatment, the $\rho$ of Sample O will eventually decrease to a steady-state value equal to that of Sample B, when the agents are completely depleted.

The compositions of (Type A) silicon oxide thermally grown at $1100{ }^{\circ} \mathrm{C}$, (Type B) as-deposited TEOS $\mathrm{SiO}_{x}$, (Type C) Type B sample annealed in $\mathrm{N}_{2}$ at $500{ }^{\circ} \mathrm{C}$ for $2 \mathrm{~h}$, and (Type D) Type $\mathrm{C}$ sample further annealed in $\mathrm{O}_{2}$ at $500{ }^{\circ} \mathrm{C}$ for $2 \mathrm{~h}$ were characterized using secondary ion-mass spectrometry (SIMS). The SIMS counts $\left[\mathrm{O}_{2}\right]$ of $\mathrm{O}_{2}$, [ $[\mathrm{Si}]$ of $\mathrm{Si},\left[\mathrm{SiO}_{3}\right]$ of $\mathrm{SiO}_{3}$, and $\left[\mathrm{SiO}_{2}\right]$ of $\mathrm{SiO}_{2}$ were extracted and the ratios $\left[\mathrm{O}_{2}\right]:[\mathrm{Si}]$ and $\left[\mathrm{SiO}_{3}\right]:\left[\mathrm{SiO}_{2}\right]$ were computed. These ratios reflect the relative abundance of oxygen in a given film. They were normalized using the respective ratios of the Type A oxide and plotted in the top inset of Fig. 2. Compared with the oxygen content in the Type A oxide, it is immediately clear that the content in the as-deposited Type $\mathrm{B}$ oxide is higher while that in the $\mathrm{N}_{2}$-annealed Type $\mathrm{C}$ oxide is lower. The reduction of the oxygen content in the Type $\mathrm{B}$ oxide provides an indirect evidence of the existence and the migration of the oxidizing agents in Sample $\mathrm{O}$ during its heat treatment

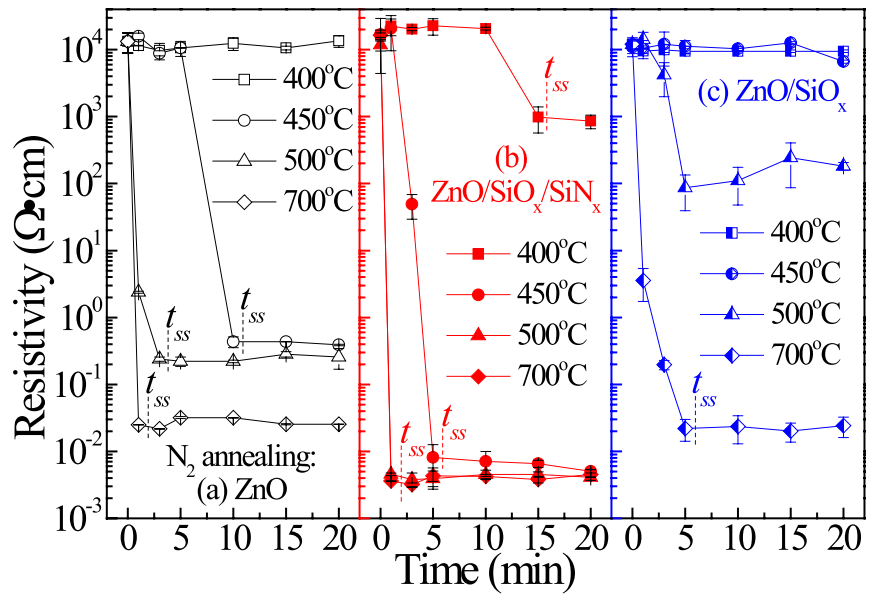

Fig. 3. Dependence of the $\rho$ on the heat-treatment time for Samples B, ON, and $\mathrm{O}$ annealed at different temperatures.

in $\mathrm{N}_{2}$. With an additional thermal treatment in $\mathrm{O}_{2}$, the depleted agents in $\mathrm{TEOS}^{\mathrm{SiO}} x$ could be replenished, as indicated by the increased oxygen content in the Type D oxide.

\section{Dependence of the $\rho$ and $t_{\mathrm{ss}}$ on the Heat-Treatment Temperature}

The time evolution of the $\rho$ was further investigated at annealing temperatures ranging from $400{ }^{\circ} \mathrm{C}$ to $700{ }^{\circ} \mathrm{C}$. Consistent with the slower kinetics of defect formation at a lower temperature, the $t_{\mathrm{ss}}$ increased with decreasing temperature for both Samples B [Fig. 3(a)] and ON [Fig. 3(b)]. The relatively constant $\rho$ for the Sample B annealed at $400{ }^{\circ} \mathrm{C}$ is a reflection of the corresponding $t_{\mathrm{Ss}}$ being longer than the $20 \mathrm{~min}$ of heat-treatment time. Again consistent with the observation that the configuration $\mathrm{ON}$ is more efficient in turning intrinsic $\mathrm{ZnO}$ into a conductor, the steady-state $\rho$ and $t_{\mathrm{ss}}$ for Sample ON are, respectively, lower and shorter than those of Sample B, at any given annealing temperature.

The behavior of the Sample O [Fig. 3(c)] was more complicated, being affected by the supply of the oxidizing agents in the oxide cover-layer. It is clear the $\rho$ (and $t_{\mathrm{ss}}$ ) for Sample O were consistently higher (and longer) that that of Sample B for any given combination of annealing temperature and time, because the oxide served not only as a barrier against the diffusion of the oxidizing species but also as a source of the oxidizing agents. With the trend obtained at $500{ }^{\circ} \mathrm{C}$ used as a reference, it can be seen that at the higher temperature of $700{ }^{\circ} \mathrm{C}$, not only was the plateau missing, the transition to a low steady-state $\rho$ was also much faster. This is an indication of the more rapid generation of defects in $\mathrm{ZnO}$ that overwhelmed the annihilation of defects by the limited supply of the oxidizing agents from the oxide. It is not surprising that the steady-state $\rho(\sim 20 \mathrm{~m} \Omega \cdot \mathrm{cm})$ of Sample $O$ was similar to that of Sample B at this higher annealing temperature, since these values were controlled only by the exchange of the oxidizing species between the ambience and the $\mathrm{ZnO}$. On the contrary, the $\rho$ stayed relatively constant at the lower annealing temperatures of $400{ }^{\circ} \mathrm{C}$ and $450{ }^{\circ} \mathrm{C}$. This is a reflection of the slower rate of defect generation that could be compensated 


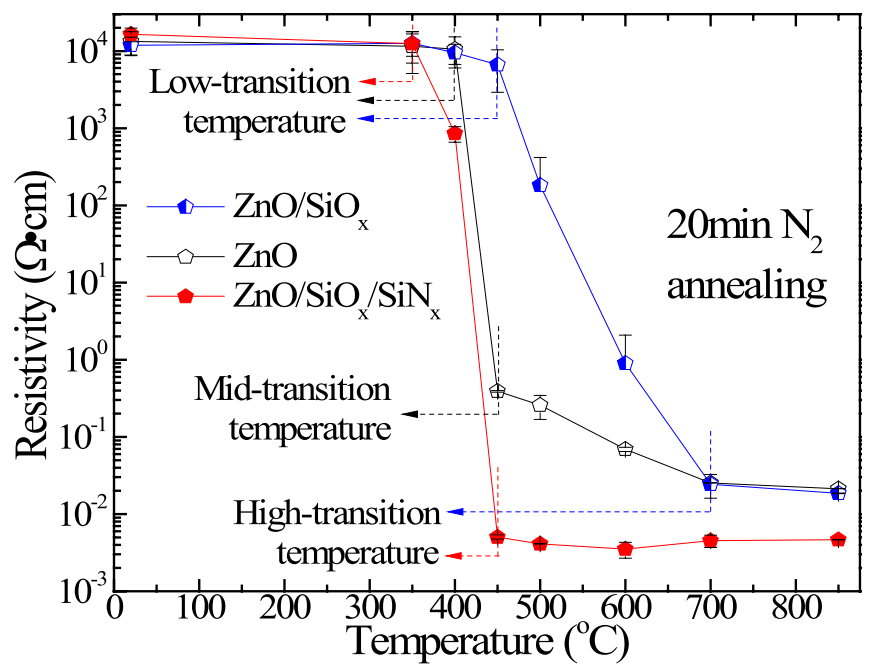

Fig. 4. Dependence of the $\rho$ on temperature for Samples B, O, and ON annealed in $\mathrm{N}_{2}$ for $20 \mathrm{~min}$.

by the supply of the oxidizing agents from the oxide. Clearly, the supply of the agents was not completely depleted during the 20-min heat treatment.

The 20-min isochronal annealing trends of the samples are summarized in Fig. 4, revealing a step-like behavior characterized by a low- and a high-transition temperature. The sharpness of the transition can be measured by the difference between the two transition temperatures, with the smallest difference, hence the sharpest transition, observed for Sample ON. Below the low-transition temperature, the $\rho$ changed little from its initial value and stayed relatively high. This temperature is the lowest $\left(350{ }^{\circ} \mathrm{C}\right)$ for Sample $\mathrm{ON}$ and the highest $\left(450{ }^{\circ} \mathrm{C}\right)$ for Sample $\mathrm{O}$. Above the high-transition temperature, a relatively constant low $\rho$ was observed. Again, this temperature is the lowest $\left(450{ }^{\circ} \mathrm{C}\right)$ for Sample $\mathrm{ON}$ and higher $\left(700{ }^{\circ} \mathrm{C}\right)$ for both Samples O and B. Within the transition region of Sample B, a more gradual reduction in the $\rho$ was observed after a rapid drop beyond a midtransition temperature $\left(450{ }^{\circ} \mathrm{C}\right)$. This could be a reflection of the different kinetics of the defect generation in these two temperature regimes.

\section{FormATION MECHANISMS OF THE ANNEALING-INDUCED DONORS IN SAMPLES

$$
\text { B, O, AND ON }
$$

\section{A. Formation Mechanisms of the Annealing-Induced Donors in the Samples $B$ and $O$}

Nitrogen, being itself a potential dopant (possibly an acceptor [1], [2]) in $\mathrm{ZnO}$, could be responsible for the low $\rho$ measured on Samples B and $\mathrm{O}$ annealed in $\mathrm{N}_{2}$. This possibility was tested by annealing the samples in argon (Ar), which is not known as a dopant in $\mathrm{ZnO}$, and eliminated when the samples gave rise to almost identical trends (Fig. 5) as in $\mathrm{N}_{2}$. Alternatively, it was more plausible to attribute the reduction in the $\rho$ to the generation of donor-type defects [6]-[9], [18], [19]. These defects, known to exhibit characteristic PL [9]-[12] spectra, were studied accordingly.

Shown in Fig. 6 are the PL spectra of Sample B after a 20-min isochronal annealing at $400{ }^{\circ} \mathrm{C}, 450{ }^{\circ} \mathrm{C}, 500{ }^{\circ} \mathrm{C}$,

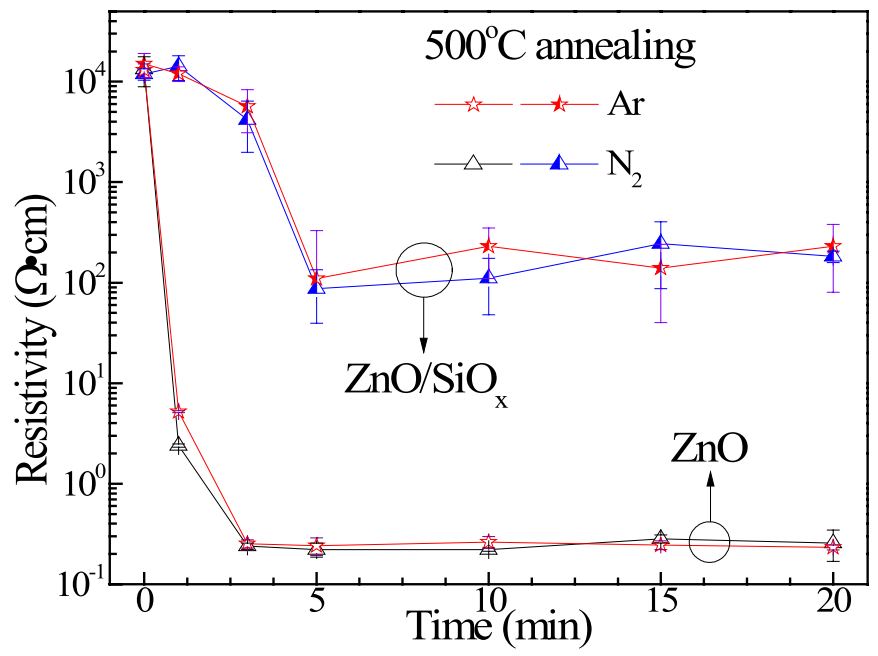

Fig. 5. Dependence of the $\rho$ on the heat-treatment time for Samples B and O annealed in $\mathrm{N}_{2}$ and $\mathrm{Ar}$.

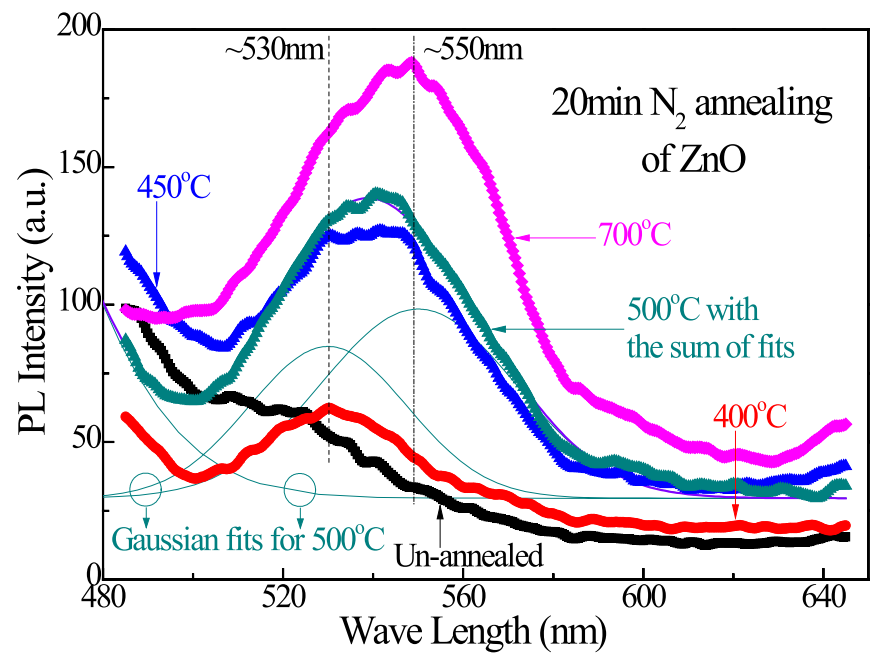

Fig. 6. PL spectra of Sample $\mathrm{B}$ annealed in $\mathrm{N}_{2}$ for $20 \mathrm{~min}$ at different temperatures. Gaussian fitting is illustrated using the spectrum of sample heattreated at $500{ }^{\circ} \mathrm{C}$

and $700{ }^{\circ} \mathrm{C}$. While there is no noticeable luminescence beyond $\sim 620 \mathrm{~nm}$, the increasing luminescence below $\sim 500 \mathrm{~nm}$ is related to that of the stoichiometric $\mathrm{ZnO}$ [9]-[12], [22], [23]. When the annealing temperature was raised, the luminescence intensity increased for all wavelengths, corresponding to an increased density of the defects. The peak also shifted to a longer wavelength, corresponding to a change of the dominant defect type. Subpeaks near 530 and $550 \mathrm{~nm}$ were revealed after the application of Gaussian fitting to the spectra. Though the correspondence between the PL peaks and their responsible defect types in $\mathrm{ZnO}$ has yet to be conclusively confirmed [9]-[12], [22], [23], there is evidence [1], [12], [24] for the respective assignment of the $\sim 530$ and $\sim 550-\mathrm{nm}$ peaks to the $\mathrm{Zn}_{\mathrm{i}}$ and $V_{\mathrm{O}}$ donor-like defects [1], [19]-[21].

The correlation between the intensities of the $\sim 530$ and $\sim 550$-nm peaks and the change in the $\rho$ is shown in Fig. 7. Compared with the intensities of the peaks of the unannealed sample, those of the sample annealed at $400{ }^{\circ} \mathrm{C}$ exhibited relatively little change, thus correlating well with the relatively 


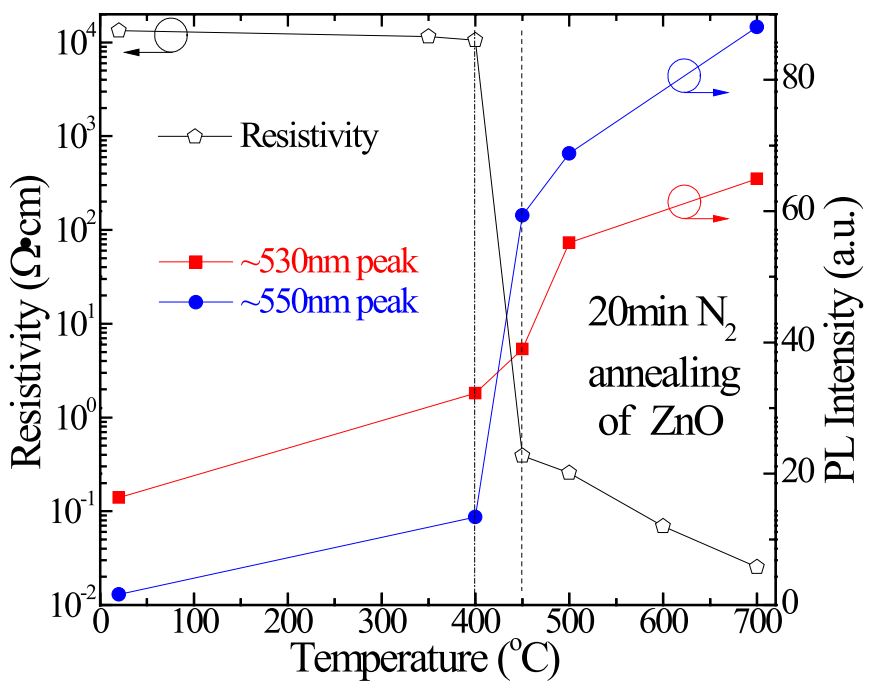

Fig. 7. Temperature dependence of the $\rho$ and the PL intensities of the $\sim 530$ and $\sim 550$-nm peaks of Sample B subjected to 20 -min isochronal annealing in $\mathrm{N}_{2}$.

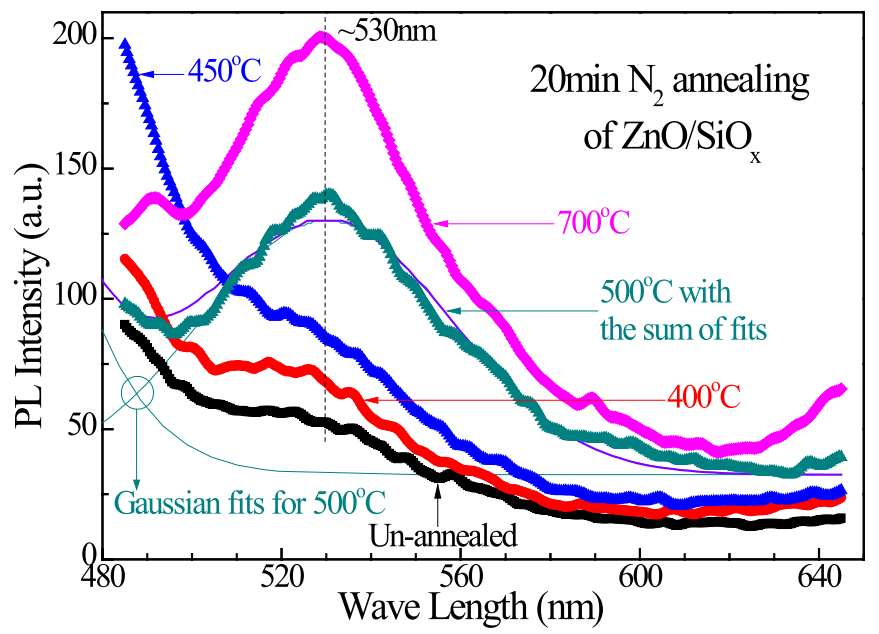

Fig. 8. PL spectra of Sample $\mathrm{O}$ annealed in $\mathrm{N}_{2}$ for $20 \mathrm{~min}$ at different temperatures.

small change in the $\rho$; the sharp increase in the intensity of the $\sim 550$-nm peak between the low-transition temperature $\left(400{ }^{\circ} \mathrm{C}\right)$ and the mid-transition temperature $\left(450{ }^{\circ} \mathrm{C}\right)$ correlates with the rapid drop in the $\rho$; while the gradual increase in the intensities of the two peaks beyond $450{ }^{\circ} \mathrm{C}$ correlates well with the slower drop in the $\rho$. The threshold of the rapid increase of the $\sim 550$-nm peak occurring at the low-transition temperature could be attributed to the lower formation energy of $V_{\mathrm{O}}$ compared with that of $\mathrm{Zn}_{\mathrm{i}}$ in a relatively oxygen poor, hence zinc rich, environment [1], [19]-[21].

In comparison, the Gaussian fitting of the PL spectra of Sample O (Fig. 8) revealed only the appearance of the peak near $530 \mathrm{~nm}$, attributed to $\mathrm{Zn}_{\mathrm{i}}$. The missing peak near $550 \mathrm{~nm}$ and attributed to $V_{O}$ is likely a result of the previously inferred supply of oxidizing agents from the cover oxide.

The correlation between the changes in the intensity of the $\sim 530$-nm peak and the $\rho$ is shown in Fig. 9. Two temperature regimes are revealed, with a relatively smaller change in

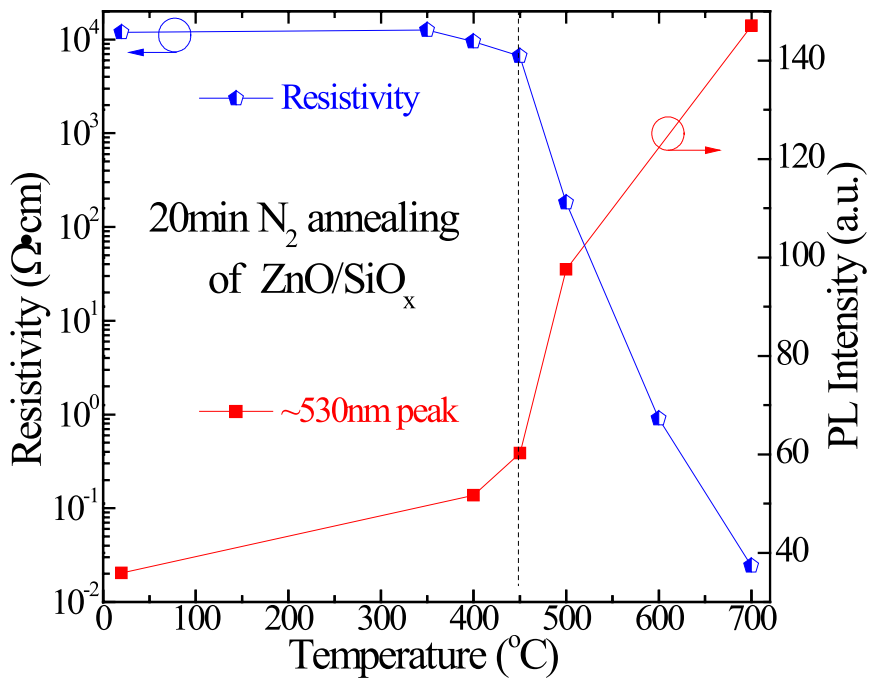

Fig. 9. Temperature dependence of the $\rho$ and the PL intensity of the $\sim 530 \mathrm{~nm}$ peak for Sample $\mathrm{O}$ subjected to 20 -min isochronal annealing in $\mathrm{N}_{2}$.

the intensity of the peak at the lower annealing temperature correlating with the smaller change in the $\rho$ and the switching at $450{ }^{\circ} \mathrm{C}$ to the relatively more rapid change in the intensity of the peak correlating well to the larger change in $\rho$ at the higher annealing temperature. An increase of $\mathrm{Zn}_{\mathrm{i}}$ population at $450{ }^{\circ} \mathrm{C}$ was consistently observed in both Samples B and O.

\section{B. Formation Mechanism of the Annealing-Induced Donors in Sample ON}

The annealing behavior of the $\rho$ of Sample ON is distinct (Figs. 1-4) from that of Samples B and O, implying a different defect generation mechanism. The possibility of Sample ON being doped with extrinsic dopants was first investigated and eliminated. Hydrogen $(\mathrm{H})$ and nitrogen $(\mathrm{N})$ were the mostly likely suspects, since PECVD $\mathrm{SiN}_{x}$ is known as a rich source of $\mathrm{H}$ [24], [25], a shallow donor in $\mathrm{ZnO}$, and $\mathrm{N}$, possibly from the $\mathrm{NH}_{3}$-containing ambience used for the PECVD of $\mathrm{SiN}_{x}$. The Samples ON annealed at different temperatures were analyzed using SIMS. The resulting temperature evolutions of the intensities of the relevant species are displayed in Fig. 10. Since $\mathrm{N}$ did not produce strong secondary-ion yield, a carbonnitrogen $(\mathrm{CN})$ complex was chosen for monitoring the $\mathrm{N}$ content in the samples. It can be seen that while the $\rho$ decreased by $\sim 10^{7}$ from the untreated sample to those annealed at temperatures beyond $450{ }^{\circ} \mathrm{C}$, the corresponding change in $\mathrm{H}$ and $\mathrm{CN}$ were less than $\sim 10^{2}$. Consequently, it is unlikely that either $\mathrm{H}$ or $\mathrm{N}$ was responsible for the observed annealing behavior of Sample ON.

With the top nitride in Sample ON replaced by a layer of 200-nm titanium sputtered at room temperature (Sample OT), the cover remained impermeable while the source of $\mathrm{H}$ and $\mathrm{N}$ from a PECVD process was eliminated. The largely identical annealing behavior of this titanium-capped Samples OT and $\mathrm{ON}$ in both $\mathrm{N}_{2}$ and $\mathrm{O}_{2}$ (the inset of Fig. 10) conclusively supported the elimination of $\mathrm{H}$ or $\mathrm{N}$ as responsible for the observed behavior of Sample ON. The generation of intrinsic 


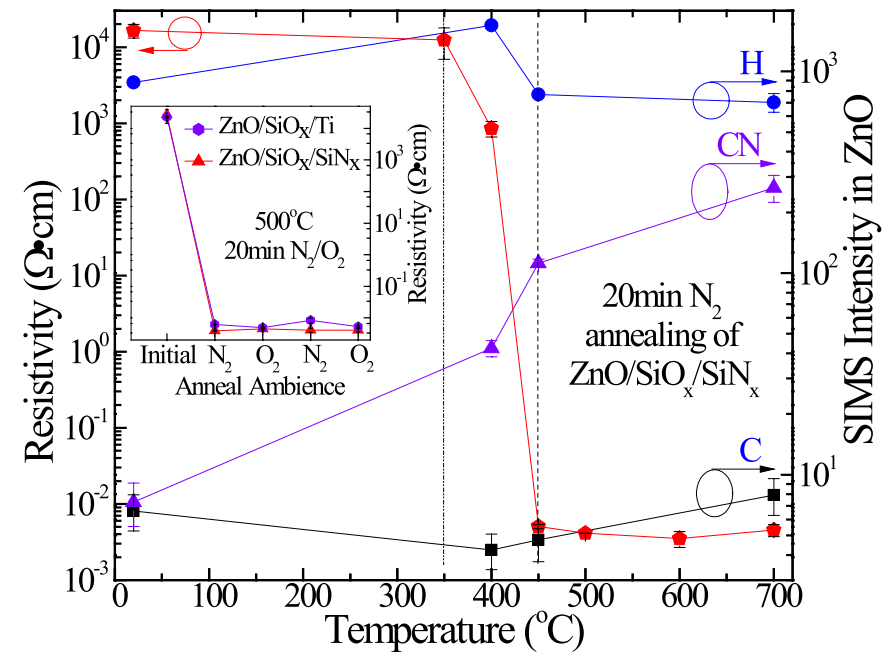

Fig. 10. Temperature dependence of the $\rho$ and SIMS intensities for Sample ON subjected to 20-min isochronal annealing in $\mathrm{N}_{2}$. Shown in the Inset is the dependence of the $\rho$ on the heat-treatment ambience for Samples OT and ON.

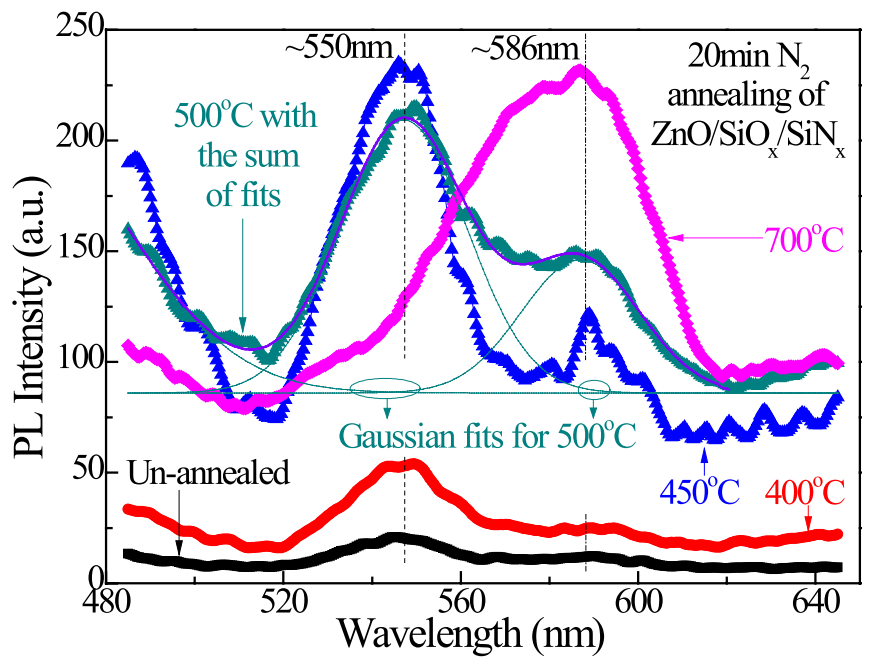

Fig. 11. PL spectra of Sample $\mathrm{ON}$ annealed in $\mathrm{N}_{2}$ for $20 \mathrm{~min}$ at different temperatures.

defects remained the more plausible cause of the observed behavior.

Two characteristic peaks near 550 and $586 \mathrm{~nm}$ could be resolved from the PL spectra of Sample ON (Fig. 11) by Gaussian fitting. While the former has already been identified with $V_{O}$, the latter is commonly assigned to the donor-like $\mathrm{Zn}_{\mathrm{O}}$ [1], [12], [24].

The temperature evolutions of the $\rho$ and the peak intensities are compared in Fig. 12. Below $350{ }^{\circ} \mathrm{C}$, the high $\rho$ is consistent with the low PL intensities, corresponding to low concentrations of $V_{\mathrm{O}}$ and $\mathrm{Zn}_{\mathrm{O}}$. The transition to low $\rho$ occurred in the temperature range between 350 and $450{ }^{\circ} \mathrm{C}$, with both intensities beginning to respond to and increase with the increase in temperature. At temperature higher than $450{ }^{\circ} \mathrm{C}$, the intensity attributed to $\mathrm{Zn}$ O kept increase while that attributed to $V_{\mathrm{O}}$ significantly reduced. Considering the almost constant $\rho$, this suggests a gradual transition of the dominant donors from both $V_{\mathrm{O}}$ and $\mathrm{Zn}_{\mathrm{O}}$ at the lower temperature to only $\mathrm{Zn}_{\mathrm{O}}$ at the higher temperature.

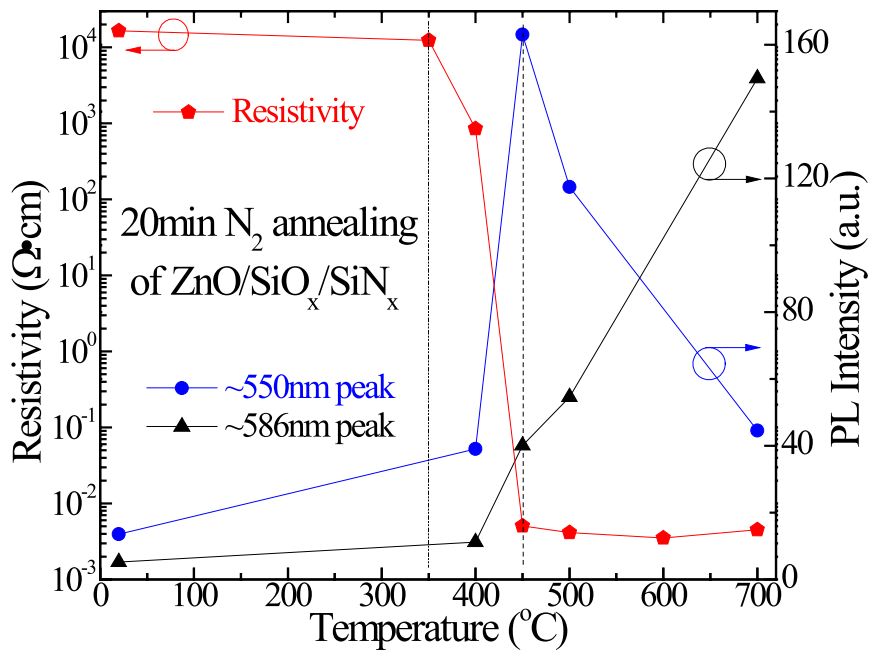

Fig. 12. Temperature dependence of the $\rho$ and the PL intensities of $\sim 550$ and $~ 586-\mathrm{nm}$ peaks for Sample ON subjected to 20-min isochronal annealing in $\mathrm{N}_{2}$.

It is reasonable to assume the intrinsic defects $V_{\mathrm{O}}$ and $\mathrm{Zn}_{\mathrm{O}}$ are stable only when $\mathrm{ZnO}$ is annealed under an impermeable seal. This was confirmed by removing the seal and subsequently annealing the samples in either $\mathrm{N}_{2}$ or $\mathrm{O}_{2}$. The $\rho$ of a bare sample annealed in $\mathrm{N}_{2}$ or $\mathrm{O}_{2}$ are, respectively, recovered, as has already been indicated by the dashed lines in Fig. 1.

\section{Annealing of Phosphorus-Doped ZnO TFTs}

Depending on the structure adopted for a TFT, such as top-gate versus bottom-gate, the channel-region could be configured differently. This channel region is then subjected to a combination of thermal schedules. Since the leakage current in the OFF-state of a TFT is largely controlled by the residual $\rho$ of the channel-region of the TFT and given the present demonstration of the sensitivities of the residual $\rho$ to the thermal processes and to the configurations of the channel, there is an implied correlation between the aggregate thermal treatment and the leakage current of the transistor. This was investigated and demonstrated.

The schematic cross section of a ZnO TFT with phosphorusdoped source and drain (S/D) regions is shown in Fig. 13 [16], [17]. The construction of the TFT started with the roomtemperature sputter-deposition of $100-\mathrm{nm} \mathrm{ZnO}$ on Corning Eagle 2000 glass. 50-nm PECVD TEOS $\mathrm{SiO}_{x}$ was subsequently deposited to form a portion of the gate-oxide. Following the patterning and etching of the active islands, a second 50-nm PECVD TEOS $\mathrm{SiO}_{x}$ was deposited to complete the formation of the gate oxide. A 200-nm indium-tin oxide (ITO) was then room-temperature sputtered and patterned to form the gate electrode. The devices were subsequently heat treated in $\mathrm{N}_{2}$ for $20 \mathrm{~min}$ at various temperatures. With the ITO gate electrode masking the channel region, phosphorus implantation was finally used to form the self-aligned $\mathrm{S} / \mathrm{D}$ regions. Even without any subsequent activation heat treatment, a resistivity of $\sim 10 \mathrm{~m} \Omega \cdot \mathrm{cm}$ was obtained after the $\mathrm{SiO}_{x}$ covering the $\mathrm{S} / \mathrm{D}$ regions were removed using an $\mathrm{SF}_{6}$-based plasma etching. With the different heat-treatments 
(a)

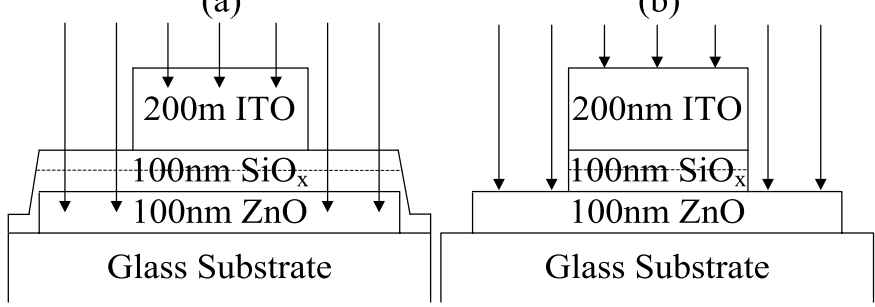

Fig. 13. Schematic cross section of the device during (a) phosphorus implantation and (b) $\mathrm{SF}_{6}$ etch.

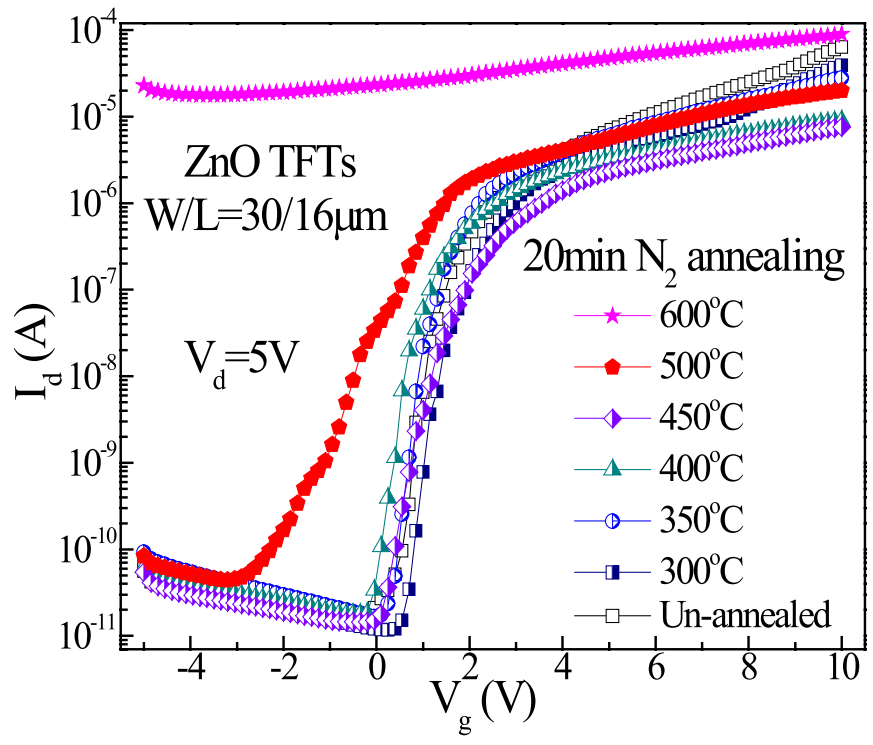

Fig. 14. Transfer characteristics of ZnO TFTs annealed in $\mathrm{N}_{2}$ for 20 min at different temperatures.

performed before the introduction of the S/D dopants and the elimination of the dopant-activation heat-treatment, any process-induced variations in the properties of the S/D regions are avoided. This makes it easier to establish a clearer correlation between the heat treatment of the channel and the leakage current of a TFT.

Compared with the transfer characteristic of the unannealed TFT (Fig. 14), those of the transistors annealed at a temperature of $450{ }^{\circ} \mathrm{C}$ or below exhibited relatively little change in the OFF-state leakage current. A rapid increase in the leakage current was measured at an annealing temperature of $500{ }^{\circ} \mathrm{C}$ and the channel essentially forms an electrical short circuit at an annealing temperature of $600{ }^{\circ} \mathrm{C}$. This behavior correlates well with the temperature dependence of the $\rho$ of Sample O (Fig. 4). More specifically, the annealing temperature must not be higher than the low-transition temperature defined in Fig. 4 to avoid excessive leakage current. This observation is generally applicable to a large variety of ZnO-based TFTs, with the recognition that the low-transition temperature is not unique but may vary with different materials and coverage configurations.

\section{CONCLUSION}

The resistivity of $\mathrm{ZnO}$ is found to depend on both the process conditions during a heat treatment and the coverage configurations of the $\mathrm{ZnO}$. The observed behavior correlates well with the change in the population of the defects in $\mathrm{ZnO}$. For a given heat-treatment condition, the resistivity of $\mathrm{ZnO}$ under an impermeable cover is found to be the lowest. The most complex heat-treatment behavior is observed for $\mathrm{ZnO}$ covered by an oxide layer, which is one of the most common configurations in the construction of a $\mathrm{ZnO}$ TFT. When a transistor with such a configuration is subjected to isochronal heat treatment in nitrogen at a temperature beyond the lowtransition temperature, the leakage current is observed to increase rather than decrease with the annealing temperature. This is found to correlate well with an increase in the defect population, hence a decrease in the resistivity of the channel region.

\section{REFERENCES}

[1] A. Janotti and C. G. Van de Walle, "Fundamentals of zinc oxide as a semiconductor," Rep. Progr. Phys., vol. 72, no. 12, p. 126501, Dec. 2009.

[2] S. J. Pearton, D. P. Norton, K. Ip, Y. W. Heo, and T. Steiner, "Recent advances in processing of ZnO," J. Vac. Sci. Technol. B, Microelectron. Nanometer Struct., vol. 22, no. 3, pp. 932-948, 2004.

[3] E. Fortunato, P. Barquinha, and R. Martins, "Oxide semiconductor thinfilm transistors: A review of recent advances," Adv. Mater. (Deerfield Beach, Fla.), vol. 24, no. 22, pp. 2945-2986, Jun. 2012.

[4] J. S. Park, W.-J. Maeng, H.-S. Kim, and J.-S. Park, "Review of recent developments in amorphous oxide semiconductor thin-film transistor devices," Thin Solid Films, vol. 520, no. 6, pp. 1679-1693, Jan. 2012.

[5] R. J. Lad, "Postdeposition annealing behavior of RF sputtered $\mathrm{ZnO}$ films," J. Vac. Sci. Technol., vol. 17, no. 4, pp. 808-811, Jul. 1980.

[6] D. K. Lee, S. Kim, M. C. Kim, S. H. Eom, H. T. Oh, and S.-H. Choi, "Annealing effect on the electrical and the optical characteristics of undoped $\mathrm{ZnO}$ thin films grown on characteristics of undoped $\mathrm{ZnO}$ thin films Si substrates by RF magnetron sputtering sputtering," J. Korean Phys. Soc., vol. 51, no. 4, pp. 1378-1382, 2007.

[7] K. Ogata, K. Sakurai, S. Fujita, S. Fujita, and K. Matsushige, "Effects of thermal annealing of $\mathrm{ZnO}$ layers grown by MBE," J. Cryst. Growth, vol. 215, pp. 312-315, Jun. 2000.

[8] T. Hirao, M. Furuta, H. Furuta, T. Matsuda, T. Hiramatsu, H. Hokari, et al., "Novel top-gate zinc oxide thin-film transistors (ZnO TFTs) for AMLCDs," J. Soc. Inf. Display, vol. 15, no. 1, pp. 17-22, 2007.

[9] L. Ke, S. C. Lai, J. D. Ye, V. L. Kaixin, and S. J. Chua, "Point defects analysis of zinc oxide thin films annealed at different temperatures with photoluminescence, Hall mobility, and low frequency noise," J. Appl. Phys., vol. 108, no. 8, pp. 084502-1-084502-6, 2010.

[10] H. S. Kang, "Annealing effect on the property of ultraviolet and green emissions of $\mathrm{ZnO}$ thin films," J. Appl. Phys., vol. 95, no. 3, pp. 1246-1250, 2004.

[11] W. S. Shi, O. Agyeman, and C. N. Xu, "Enhancement of the light emissions from zinc oxide films by controlling the post-treatment ambient," J. Appl. Phys., vol. 91, no. 9, pp. 5640-5644, 2002.

[12] X. Q. Wei, B. Y. Man, M. Liu, C. S. Xue, H. Z. Zhuang, and C. Yang, "Blue luminescent centers and microstructural evaluation by XPS and Raman in $\mathrm{ZnO}$ thin films annealed in vacuum, $\mathrm{N}_{2}$ and $\mathrm{O}_{2}$," Phys. $B$, Condensed Matter, vol. 388, nos. 1-2, pp. 145-52, Jan. 2007.

[13] S. T. Meyers, J. T. Anderson, C. M. Hung, J. Thompson, J. F. Wager, and D. A. Keszler, "Aqueous inorganic inks for low-temperature fabrication of ZnO TFTs," J. Amer. Chem. Soc., vol. 130, no. 51, pp. 17603-17609, Dec. 2008.

[14] B. S. Ong, C. Li, Y. Li, Y. Wu, and R. Loutfy, "Stable, solutionprocessed, high-mobility $\mathrm{ZnO}$ thin-film transistors," J. Amer. Chem. Soc., vol. 129, no. 10, pp. 2750-2751, Mar. 2007.

[15] L. J. Brillson and Y. Lu, "ZnO Schottky barriers and Ohmic contacts," J. Appl. Phys., vol. 109, no. 12, pp. 121301-1-121301-33, 2011.

[16] Z. Ye and M. Wong, "Characteristics of thin-film transistors fabricated on fluorinated zinc oxide," IEEE Electron Device Lett., vol. 33, no. 4 pp. 549-551, Apr. 2012.

[17] Z. Ye and M. Wong, "Investigation of phosphorus and arsenic as dopants in polycrystalline thin films of zinc oxide," J. Appl. Phys., vol. 113, no. 2, pp. 024506-1-024506-6, 2013. 
[18] G. Z. Xing, B. Yao, C. X. Cong, T. Yang, Y. P. Xie, B. H. Li, et al., "Effect of annealing on conductivity behavior of undoped zinc oxide prepared by RF magnetron sputtering," J. Alloys Compounds, vol. 457, nos. $1-2$, pp. $36-41$, Jun. 2008

[19] D. Look, G. Farlow, P. Reunchan, S. Limpijumnong, S. Zhang, and K. Nordlund, "Evidence for native-defect donors in n-type ZnO," Phys. Rev. Lett., vol. 95, no. 22, pp. 1-4, Nov. 2005.

[20] F. Selim, M. Weber, D. Solodovnikov, and K. Lynn, "Nature of native defects in ZnO," Phys. Rev. Lett., vol. 99, no. 8, pp. 085502-1-085502-4, Aug. 2007.

[21] D. C. Look and J. W. Hemsky, "Residual native shallow donor in ZnO," Phys. Rev. Lett., vol. 82, no. 12, pp. 2552-2555, 1999.

[22] S.-H. Jeong, B.-S. Kim, and B.-T. Lee, "Photoluminescence dependence of $\mathrm{ZnO}$ films grown on $\mathrm{Si}(100)$ by radio-frequency magnetron sputtering on the growth ambient," Appl. Phys. Lett., vol. 82, no. 16, p. 2625, 2003.

[23] B. Lin, Z. Fu, and Y. Jia, "Green luminescent center in undoped zinc oxide films deposited on silicon substrates," Appl. Phys. Lett., vol. 79, no. 7, pp. 943-945, 2001.

[24] C. Wu, H. Hsieh, C. Chien, and C. Wu, "Self-aligned top-gate coplanar In-Ga-Zn-O,” J. Display Technol., vol. 5, no. 12, pp. 515-519, 2009.

[25] D. H. Kang, I. Kang, S. H. Ryu, and J. Jang, "Self-aligned coplanar a-IGZO TFTs and application to high-speed circuits," IEEE Electron Device Lett., vol. 32, no. 10, pp. 1385-1387, Oct. 2011.

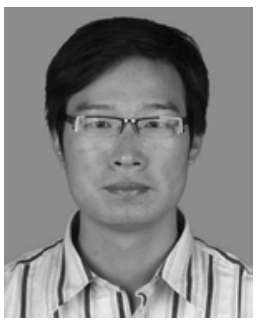

Lei Lu received the B.S. and M.S. degrees in microelectronics from Soochow University, Jiangsu, China, in 2007 and 2010, respectively. He is currently pursuing the Ph.D. degree with the Department of Electronic and Computer Engineering, Hong Kong University of Science and Technology, Hong Kong.

His current research interests include the annealing behaviors of $\mathrm{ZnO}$-based metal oxide materials and the fabrication and reliability study of TFTs based on metal oxides.

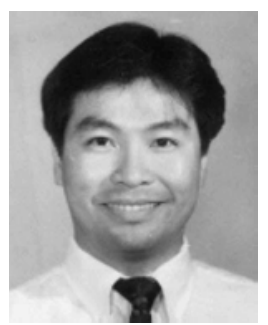

Man Wong (S'83-M'84-SM'00) received the B.S and M.S. degrees in electrical engineering from the Massachusetts Institute of Technology, Cambridge, MA, USA, in 1982 and 1984, respectively, and the $\mathrm{Ph} . \mathrm{D}$. degree in electrical engineering from the Center for Integrated Systems, Stanford University, Stanford, CA, USA.

His research interests include micro-fabrication technology, device structure and material, physics and technology of thin-film transistor, organic lightemitting diode display technology, and modeling and implementation of integrated microsystems. 